\title{
ON A GOLAY-SHAPIRO-LIKE SEQUENCE
}

\author{
Jean-Paul Allouche \\ Dedicated to the memory of Pierre Liardet
}

\begin{abstract}
A recent paper by P. Lafrance, N. Rampersad, and R. Yee studies the sequence of occurrences of 10 as a scattered subsequence in the binary expansion of integers. They prove in particular that the summatory function of this sequence has the "root $N$ " property, analogously to the summatory function of the Golay-Shapiro sequence. We prove here that the root $N$ property does not hold if we twist the sequence by powers of a complex number of modulus one, hence showing a fundamental difference with the Golay-Shapiro sequence.
\end{abstract}

\section{Communicated by Reinhard Winkler}

\section{Introduction}

In the paper [7] the authors study a sequence $\left(i_{n}\right)_{n \geq 0}$ involving the number $i n v_{2}(n)$ of inversions in the binary expansion of the integer $n$, i.e., the number of occurrences of 10 as a scattered subsequence of the binary representation of the integer $n$. More precisely, defining $i_{n}:=(-1)^{i n v_{2}(n)}$ they prove in particular the following result.

TheOREM 1 (Theorem 2 in [7]). There exists a bounded, continuous, nowhere differentiable, 1-periodic function $G$ such that

$$
S(N):=\sum_{0 \leq n \leq N} i_{n}=\sqrt{N} G\left(\log _{4} N\right) .
$$

This shows that the behavior of the summatory function of sequence $\left(i_{n}\right)_{n \geq 0}$ is quite similar to the behavior of the summatory function of the Golay-Shapiro sequence (see [4, 3]). Recall that the \pm 1 Golay-Shapiro sequence $\left(a_{n}\right)_{n \geq 0}$ is

2010 Mathematics Subject Classification: Primary: 11K16; Secondary: $11 B 85$.

Keywords: binary expansion, digital sequence, Rudin-Shapiro sequence, summatory function.

Partially supported by the ANR project "FAN" (Fractals et Numération), ANR-12-IS01-0002. 
defined by $a_{n}=(-1)^{w_{n}}$, where $w_{n}$ counts the number of possibly overlapping 11's in the binary expansion of the integer $n$. This sequence can also be defined by $a_{0}=1$, and for all $n \geq 0$, the recurrence relations $a_{2 n}=a_{n}$ and $a_{2 n+1}=(-1)^{n} a_{n}$ (see [2]). It is then natural to ask, as the authors of [7] do, whether the sequence $\left(i_{n}\right)_{n \geq 0}$ satisfies the fundamental "root $N$ " property of the Golay-Shapiro sequence, namely

$$
\sup _{\theta \in \mathbb{R}}\left|\sum_{0 \leq n \leq N} a_{n} e^{2 i \pi n \theta}\right|=O(\sqrt{N}) .
$$

This question is furthermore justified not only by the fact that $\left(a_{n}\right)_{n>0}$ admits a "digital" representation as $\left(i_{n}\right)_{n \geq 0}$ does (namely $a_{n}=(-1)^{u_{n}}$, where $u_{n}$ is the number of possibly overlapping 11's in the binary expansion of $n$ ), but also by the fact that many other "digital" sequences have the root $N$ property (see [1]). The purpose of this paper is to prove that the sequence $\left(i_{n}\right)_{n \geq 0}$ does not satisfy the root $N$ property.

REMaRK 1. The Golay-Shapiro sequence is also called the Rudin-Shapiro or the Shapiro-Rudin sequence. Since Rudin [8, p. 855] acknowledges Shapiro's priority of [9], and since [9] and [6] appeared the same year, the sequence should indeed be called the "Golay-Shapiro sequence". Note that the fact that this sequence appears in a somewhat disguised form in the paper of Golay [6] can be found in the article of Brillhart and Morton [5] where they write that Odlyzko pointed out to them [6, bottom of p. 469].

\section{Preliminary results}

First we recall a property of sequence $\left(i_{n}\right)_{n \geq 0}$ given in [7].

Proposition 1 (Proposition 1 of [7]). The sequence $\left(i_{n}\right)_{n \geq 0}$ satisfies $i_{0}=1$ and the following recurrence relations: for all $n \geq 0$,

$$
i_{2 n+1}=i_{n}, \quad i_{4 n}=i_{n}, \quad i_{4 n+2}=-i_{2 n} .
$$

This proposition implies the following result on the summatory function of $\left(i_{n} z^{n}\right)_{n \geq 0}$.

Proposition 2. Let $z$ be a complex number. Define the sum $T(N, z)$ by

$$
T(N, z):=\sum_{0 \leq n \leq 2^{N}-1} z^{n}\left(\begin{array}{c}
i_{n} \\
i_{2 n}
\end{array}\right) .
$$


Then we have

$$
T(N+1, z)=\left(\begin{array}{cc}
z & 1 \\
1 & -z
\end{array}\right) T\left(N, z^{2}\right)
$$

P r o o f. Separating even and odd indices in $T(N+1, z)$ and using Proposition 1 yields

$$
\begin{aligned}
T(N+1, z) & =\sum_{0 \leq 2 n \leq 2^{N+1}-1} z^{2 n}\left(\begin{array}{l}
i_{2 n} \\
i_{4 n}
\end{array}\right)+\sum_{0 \leq 2 n+1 \leq 2^{N+1}-1} z^{2 n+1}\left(\begin{array}{l}
i_{2 n+1} \\
i_{4 n+2}
\end{array}\right) \\
& =\sum_{0 \leq n \leq 2^{N}-1} z^{2 n}\left(\begin{array}{ll}
0 & 1 \\
1 & 0
\end{array}\right)\left(\begin{array}{c}
i_{n} \\
i_{2 n}
\end{array}\right)+\sum_{0 \leq n \leq 2^{N}-1} z^{2 n+1}\left(\begin{array}{cc}
1 & 0 \\
0 & -1
\end{array}\right)\left(\begin{array}{c}
i_{n} \\
i_{2 n}
\end{array}\right) \\
& =\left(\begin{array}{cc}
z & 1 \\
1 & -z
\end{array}\right) T\left(N, z^{2}\right) .
\end{aligned}
$$

It happens that a single common transformation gives a simpler form for all matrices

$$
\left(\begin{array}{cc}
z & 1 \\
1 & -z
\end{array}\right)
$$

Proposition 3. Let $i$ be a square root of -1 and $P$ be the matrix defined by

Then

$$
P:=\left(\begin{array}{cc}
1 & 1 \\
i & -i
\end{array}\right) \text {. }
$$

$$
P^{-1}=\frac{1}{2}\left(\begin{array}{cc}
1 & -i \\
1 & i
\end{array}\right) \quad \text { and } \quad P^{-1}\left(\begin{array}{cc}
z & 1 \\
1 & -z
\end{array}\right) P=\left(\begin{array}{cc}
0 & z-i \\
z+i & 0
\end{array}\right) .
$$

P r o of. Straightforward.

Proposition 4. Let $M(z)=\left(\begin{array}{cc}z & 1 \\ 1 & -z\end{array}\right)$. Then we have

$$
\sum_{0 \leq n \leq 2^{2 N}-1} i_{n} z^{n}=\frac{1}{2}\left(c_{N}(1-i)+d_{N}(1+i)\right),
$$

where

$$
c_{N}=\prod_{0 \leq k \leq N-1}\left(z^{4^{k}}-i\right)\left(z^{2.4^{k}}+i\right) \quad \text { and } \quad d_{N}=\prod_{0 \leq k \leq N-1}\left(z^{4^{k}}+i\right)\left(z^{2.4^{k}}-i\right) .
$$

P r o o f. Using Proposition 2 we have

$$
T(N+1, z)=M(z) T\left(N, z^{2}\right)=M(z) M\left(z^{2}\right) T\left(N-1, z^{4}\right)=\cdots
$$


hence, starting from $T(N, z)$ yields

$$
T(N, z)=M(z) M\left(z^{2}\right) \cdots M\left(z^{2^{N-1}}\right) T\left(0, z^{2^{N}}\right) .
$$

Replacing $N$ by $2 N$, defining $\widetilde{M}(z):=\left(\begin{array}{cc}0 & z-i \\ z+i & 0\end{array}\right)$, and using Proposition 3 gives

$$
\begin{aligned}
T(2 N, z) & =\left(P \widetilde{M}(z) P^{-1}\right) \cdots\left(P \widetilde{M}\left(z^{2^{2 N-1}}\right) P^{-1}\right) T\left(0, z^{2^{2 N}}\right) \\
& =P \widetilde{M}(z) \cdots \widetilde{M}\left(z^{2^{2 N-1}}\right) P^{-1} T\left(0, z^{2^{2 N}}\right) \\
& =P\left(\begin{array}{cc}
0 & z-i \\
z+i & 0
\end{array}\right) \cdots\left(\begin{array}{cc}
0 & z^{2^{2 N-1}}-i \\
z^{2^{2 N-1}}+i & 0
\end{array}\right) P^{-1} T\left(0, z^{2^{2 N}}\right) .
\end{aligned}
$$

Grouping the matrices in the last equality pairwise and noting that

$$
\left(\begin{array}{cc}
0 & z-i \\
z+i & 0
\end{array}\right)\left(\begin{array}{cc}
0 & z^{2}-i \\
z^{2}+i & 0
\end{array}\right)=\left(\begin{array}{cc}
(z-i)\left(z^{2}+i\right) & 0 \\
0 & (z+i)\left(z^{2}-i\right)
\end{array}\right)
$$

we obtain

$$
T(2 N, z)=P\left(\begin{array}{cc}
c_{N} & 0 \\
0 & d_{N}
\end{array}\right) P^{-1} T\left(0, z^{2^{2 N}}\right)
$$

with

$$
c_{N}=\prod_{0 \leq k \leq N-1}\left(z^{4^{k}}-i\right)\left(z^{2.4^{k}}+i\right)
$$

and

$$
d_{N}=\prod_{0 \leq k \leq N-1}\left(z^{4^{k}}+i\right)\left(z^{2 \cdot 4^{k}}-i\right)
$$

Since for any complex number $Z$ we have

$$
T(0, Z)=Z^{0}\left(\begin{array}{l}
i_{0} \\
i_{0}
\end{array}\right)=\left(\begin{array}{l}
1 \\
1
\end{array}\right)
$$

Equality (1) can be rewritten as

$$
T(2 N, z)=\frac{1}{2}\left(\begin{array}{c}
c_{N}(1-i)+d_{N}(1+i) \\
c_{N}(1+i)+d_{N}(1-i)
\end{array}\right) .
$$

So that we have

$$
\sum_{0 \leq n \leq 2^{2 N}-1} i_{n} z^{n}=\frac{1}{2}\left(c_{N}(1-i)+d_{N}(1+i)\right) .
$$




\section{ON A GOLAY-SHAPIRO-LIKE SEQUENCE}

\section{The main result}

Now we state and prove our main theorem.

TheOrem 2. Define $j:=e^{2 i \pi / 3}$. Let $z$ be a complex number such that there exists an integer $r>0$ with $z^{4^{r}}=j$. Then there exists a positive constant $C$ (depending on $z$ ) such that, for all $N$ large enough, the following inequality holds:

$$
\left|\sum_{0 \leq n \leq 2^{2 N}-1} i_{n} z^{n}\right| \geq C(2+\sqrt{3})^{N} .
$$

In particular, for such a complex number $z,\left|\sum_{0 \leq k \leq n-1} i_{k} z^{k}\right|$ is not $O(\sqrt{n})$.

P r o o f. If $z^{4^{r}}=j$, then, for all $d \geq r$, we clearly have

$$
z^{4^{d}}=\left(z^{4^{r}}\right)^{4^{r-d}}=j
$$

(note that $j^{3}=1$ hence $j^{4}=j$ ). Thus, since

$$
(j-i)\left(j^{2}+i\right)=2-\sqrt{3} \text { and }(j+i)\left(j^{2}-i\right)=2+\sqrt{3},
$$

we have

$$
\begin{aligned}
c_{N} & =\prod_{0 \leq k \leq N-1}\left(z^{4^{k}}-i\right)\left(z^{2 \cdot 4^{k}}+i\right) \\
& =\prod_{0 \leq k \leq r-1}\left(z^{4^{k}}-i\right)\left(z^{2 \cdot 4^{k}}+i\right) \prod_{r \leq k \leq N-1}(j-i)\left(j^{2}+i\right) \\
& =A_{z}(2-\sqrt{3})^{N}
\end{aligned}
$$

for some constant

$$
A_{z}=\prod_{0 \leq k \leq r-1}\left(z^{4^{k}}-i\right)\left(z^{2.4^{k}}+i\right)\left[(j-i)\left(j^{2}+i\right)\right]^{-1} \neq 0 .
$$

Similarly $d_{n}=B_{z}(2+\sqrt{3})^{N}$ for some nonzero constant $B_{z}$.

Finally, this gives

$$
\begin{aligned}
\left|\sum_{0 \leq n \leq 2^{2 N}-1} i_{n} z^{n}\right| & =\frac{\left|d_{N}(1+i)+(1-i) c_{N}\right|}{2} \\
& \geq \frac{\left|(2+\sqrt{3})^{N}\right| B_{z}|-| A_{z}\left|(2-\sqrt{3})^{N}\right|}{\sqrt{2}} \\
& \geq C_{z}(2+\sqrt{3})^{N}
\end{aligned}
$$

for some positive constant $C_{z}$ and $N$ large enough. 


\section{JEAN-PAUL ALLOUCHE}

REMARK 2. Actually Theorem 2 above shows that there exists a dense set of real numbers $\theta$ such that

$$
\left|\sum_{0 \leq n \leq N-1} i_{n} e^{2 i \pi n \theta}\right| \text { is not } O(\sqrt{N}) .
$$

ACKnowledgements. We thank the referee for very useful remarks.

\section{REFERENCES}

[1] ALlOUChE, J.-P.-LIARDET, P.: Generalized Rudin-Shapiro sequences, Acta Arith. 60 (1991) 1-27.

[2] BRILlharT, J.-CARLITZ, L.: Note on the Shapiro polynomials, Proc. Amer. Math. Soc. 25 (1970) 114-118.

[3] BRILlHART, J.-ERDŐS, P.-MORTON, P.: On sums of Rudin-Shapiro coefficients, II, Pacific J. Math. 107 (1983) 39-69.

[4] BRILlHART, J.-MORTON, P.: Über Summen von Rudin-Shapiroschen Koeffizienten, Illinois J. Math. 22 (1978) 126-148.

[5] BRILlhART, J.-MORTON, P.: A case study in mathematical research: The GolayRudin-Shapiro sequence, Amer. Math. Monthly 103 (1996) 854-869.

[6] GOLAY, M. J. E.: Statistic multislit spectrometry and its application to the panoramic display of infrared spectra, J. Optical Soc. America 41 (1951) 468-472.

[7] LAFRANCE, P.-RAMPERSAD, N.-YEE, R.: Some properties of a Rudin-Shapiro-like sequence, Adv. Appl. Math. 63 (2015) 19-40.

[8] RUDIN, W.: Some theorems on Fourier coefficients, Proc. Amer. Math. Soc. 10 (1959) $855-859$.

[9] SHAPIRO, H. S.: Extremal Problems for Polynomials and Power Series, Thesis (M. S.), Massachusetts Institute of Technology, Department of Mathematics, 1951, available at: http://dspace.mit.edu/handle/1721.1/12198

Received March 25, 2016

Accepted July 25, 2016

\author{
Jean-Paul Allouche \\ CNRS, Institut de Mathématiques \\ de Jussieu-PRG \\ Université Pierre et Marie Curie, \\ Case 247 \\ 4 Place Jussieu \\ F-75252 Paris Cedex 05 \\ FRANCE \\ E-mail: jean-paul.allouche@imj-prg.fr
}

